\title{
Single Level Tunneling Model for Molecular Junctions: Evaluating the Simulation Methods
}

\author{
Esther Martine, ${ }^{a}$ Xianneng Song, ${ }^{b} \mathrm{Xi} \mathrm{Yu}^{\ddagger^{*}}$ and Wenping $\mathrm{Hu}^{\ddagger}$
}

Single level tunneling model has been the most popular model system in both the experimental and theoretical study of molecular junctions. We performed a detailed simulation study on the performance of the single level tunneling model in analyzing the charge transport mechanism of molecular junctions. Three different modeling methods, including the numerical integration of the Landauer formula and two approximated analytical formulas that are extensively used for extracting key transport parameters from current-voltage (I-V) characteristics, i.e. the energy offset and the coupling between molecule and electrode, were compared and evaluated for their applicability. The simulation of I-V plots shows that the applicability of the two approximated analytical models is energy offset and coupling strength dependent. Model fitting based on the three methods performed on experimental data attained from representative literature papers revealed that the two approximated analytical methods are neither suitable for the situation of small coupling strength and low energy offset, and they also deviated from the exact results at high bias. We finally provided a phase map of the applicability of different modeling methods as a guide for their proper usage in charge transport study in molecular devices.

KEY TERMS: Molecular Junction, Tunneling Transport, Single level model, Landauer Formula 
0.1

\section{Introduction}

Molecular electronics ${ }^{1-3}$ enables miniaturization of the molecular devices that has essential applications in nanosized electronics circuits, and offered opportunity to study fundamentals of charge transport mechanisms in molecular scale. In molecular junctions ${ }^{4-6}$ two transport mechanisms that broadly studied are, tunneling ${ }^{7-10}$ and hopping. ${ }^{11-13}$ Tunneling mechanism is the mechanism where electron coherently tunnel through energy offset of a molecule. It has been observed that the coherent tunneling depends on the interaction between the molecule and the contact electrode, as well as energy offset between the Fermi energy level of the electrode and the molecule orbital (sometimes also referred as tunneling barrier though not very accurate). Tunneling mechanism can be modelled based on various suppositions, while the most commonly used is single level model (SLM) in both experimental and theoretical studies. In this model, single electroactive state is sandwiched between two electrodes with energy level offset to the Fermi level of the electrodes and electronic coupling to the electronic states of the electrode (figure 1). Experimentally single state junction has been achieved by attaching electroactive group like ferrocene or conjugate organic molecules as the single electronic active state with alkane molecule as insert molecular linker to adjust the coupling of the electronic state with electrode. Function and special transport behaviors have been observed like, rectification ${ }^{14}$, Coulomb blockade and Kondo effect ${ }^{15}, 16$ through utilization of experimental techniques such as scanning tunneling microscope (STM), mechanical controllable break junctions (MC-BJ) ${ }^{17}$, conducting probe atomic force microscope (CP-AFM) ${ }^{18}$ and liquid junction techniques like eutectic indium gallium (EGaln) or mercury drop junctions ${ }^{19,} 20$. Theoretical analysis based on SLM depends on the mathematical modeling of the experimental I-V plot where coupling strength $(\Gamma)$ and energy offset $\left(\varepsilon_{0}\right)$ can be obtained and cross compared to spectroscopy measurement like ultraviolet photoelectron spectroscopy (UPS) or ab initio calculation ${ }^{21}$ The currentvoltage (I-V) relation in single level model is described by Landauer formula ( $\underline{E q} 1$ ) with single level transmission function ${ }^{9,11}$

$I=\frac{2 e^{2}}{h} \int_{-\infty}^{\infty} d E \operatorname{Tr}(E)\left[f_{L}(E)-f_{R}(E)\right]$

$f_{L}(E)$ and $f_{R}(E)$ Fermi function at left and right electrode respectively, given by

$f_{L, R}(E)=\frac{1}{\left.1+\exp \left(\left(E-\mu_{L, R}\right) / k T\right)\right)}$
And $\operatorname{Tr}(E)$ is defined as Transmission function given by,

$\operatorname{Tr}(E)=D_{\varepsilon}(E) 2 \pi \frac{\Gamma_{L} \Gamma_{R}}{\Gamma}$

Where $\Gamma=\Gamma_{L}+\Gamma_{R}, \Gamma_{L}$ and $\Gamma_{R}$ are coupling strength of the electronic state with electrode and $D_{\varepsilon}(\mathrm{E})$, is the broadened electron density of states by the interaction of the energy level $\varepsilon$ and the electrode, which is

$D_{\varepsilon}(E)=\frac{\frac{\Gamma}{2 \pi}}{(E-\varepsilon)^{2}+\left(\frac{\Gamma}{2}\right)^{2}}$

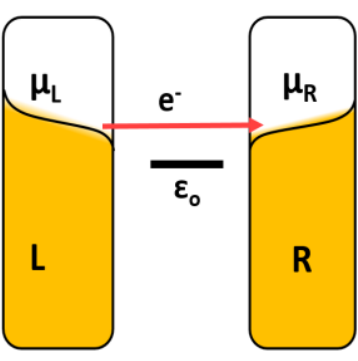

$\mathrm{V}=\mathbf{0}$

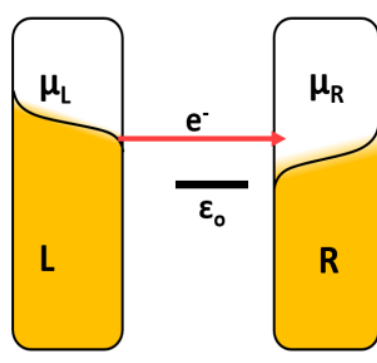

$\mathrm{V}>0$
Figure 1: schematic illustration of single level system

The integration in Eq 1 is apparently not easy to perform due to the complex mathematical form of Fermi function and density of state. There is no simple analytical relation for current and voltage to be used for experimental data analysis. Various approximated modeling methods have been invented and applied to fit the experimental I-V results to extract transport parameters like coupling strength $(\Gamma)$ and energy offset $\left(\varepsilon_{0}\right)^{22,23}$. The first one is to perform numerical integration of Eq 1. By decreasing the step size in the numerical integration, a high accuracy can be achieved. On other hand, when the Fermi broadening is small relative to the density of states, or if at low temperature, the Fermi function in Eq 1 can be approximated by a step function, and an analytical expression of $\mathrm{I}-\mathrm{V}$ can be obtained as ${ }^{11,22}$

$I=\frac{2 e^{2}}{h} \frac{\Gamma_{L} \Gamma_{L_{R}}}{\Gamma}\left(\tan ^{-1} \frac{\Delta \varepsilon+e V / 2}{\Gamma}-\tan ^{-1} \frac{\Delta \varepsilon-e V / 2}{\Gamma}\right)$

A further simplification was proposed based on Eq 2 by Baldea 22,23 under the condition $\Gamma \ll \Delta \varepsilon$ and low bias (not greater than transition voltage), which gave

$I=\frac{2 e^{2}}{h} \Gamma_{L} \Gamma_{R}\left(\frac{e V}{\Delta \varepsilon^{2}-\left(\frac{e V}{2}\right)^{2}}\right)$ 
Where $\Delta \varepsilon$ energy offset and $\Gamma=\left(\Gamma_{\mathrm{L}}+\Gamma_{\mathrm{R}}\right) / 2$, eV is the applied bias measured in electron volts. And the finite resistance arises at the interface between electrode and molecule, expressed as quantum conductance $2 \mathrm{e}^{2} / h$. We can see, more and more approximations were applied from Eq 1 to Eq 3, so the applicability should in principle reduce from Eq 1 to Eq 3 accordingly.

The current voltage (I-V) analysis using Eq 1 as the first recognized SLM expression in tunneling junction analysis achieves to unveil various features of the molecular junctions. For example, Luka-Guth et al studied the role played by the solvent in electronic transport in molecular junction, the junction analysis help them to make a reasonable conclusion on differentiated solvent conductance with that of molecular junction ${ }^{17}$. On the other hand, Zotti et al revealed the effect of anchoring group in molecular junction by examining the influence of moleculemetal contact interaction in molecule junction ${ }^{24}$. Tunneling junction analysis based on simplified I-V relation have been currently growing in popularity due to their simplifications. For example, Emanuel and coworkers ${ }^{25}$ reported their study using Eq 2 in junction analysis to compare the effect of thiols and isocyanide as anchoring group in 1,4 benzene dithiols and 1,4 benzene diisocyanide molecular junction. The conclusion drawn is the same as Zotti et $\mathrm{al}^{24}$, though the molecule differ by one benzene ring, but the extracted key parameters appear to be significantly different. Eq 3 has also attracted many studies, and it closely related to another popular method in characterizing the energy offset in the molecular junctions, i.e. transition voltage spectroscopy (TVS) ${ }^{26,27}$. TVS enables determination of transition voltage $\left(\mathrm{V}_{\text {trans }}\right)$ in a Fowler-Nordheim plot (F-N). However, as revealed by Vilan et al later on, $\mathrm{V}_{\text {trans }}$ is actually not a sign of the F-N transition, rather it is a mathematical sign of none linearity of I-V plot. Baldea derived an approximated relation between $\mathrm{V}_{\text {trans }}$ and energy offset based on Eq 3, i.e.e $V_{\text {trans }}=2 \Delta \varepsilon / \sqrt{3}$, which later on was found to be effective and acceptable method in analyzing molecular energy levels. Frisbie and coworkers ${ }^{18,23,28,29}$ have extensively used this method to examine molecular junction of different molecules on metal contacts ( $\mathrm{Ag}, \mathrm{Au}$ and $\mathrm{Pt}$ ) and explore fascinating junction characteristics and features. However, when making comparison of extracted parameters with other methods like Eq 2, we observed substantial difference in energy offset $\left(\varepsilon_{0}\right)$ for oligophenylene dithiols (OPD1) ${ }^{28}$ analyzed using Eq 3 (Frisbie et al. results) and Eq 2 (Emanuel et al results ${ }^{25}$ ) which was $0.87 \mathrm{eV}$ and $0.26 \mathrm{eV}$ respectively on $\mathrm{Au} / \mathrm{Au}$ electrode. The observed variation can be attributed to different factors, such as adopted method for I-V measurement, among which the most important one is the applicability of SLM modeling methods. In addition, SLMs have also been applied to thermoelectricity studies, such like extracting the Seebeck Coefficient, which can help to understand electronic structure of the molecular junction, i.e., Fermi level of the electrodes with respect to the HOMO or LUMO levels of the molecules, through determining the type of charge carriers (either $\mathrm{p}$ - or n-type) ${ }^{30}$.

SLM is the method that has triumphed theoretical I-V studies as powerful and valuable analyzing methods in exploring tunneling transport in molecular junction. The theoretical modeling of $\mathrm{I}-\mathrm{V}$ is promising to understand energy level in charge transport at the molecular junction, which also relies crucially on our proficiency of utilizing these modeling methods. However, the applicability and accuracy of using these models have not been well evaluated. Indeed, different single level mathematical methods also rendered significant variation in extracted transport parameters. We thus developed the interest of detailed examination of condition and limitation of their application in modeling I-V response of the junction. This work provides extensive study on SLM methods, including the numerical integration of Landauer formula (Eq 1), and other two analytical tunneling models, Eq 2 and Eq 3, believing that the main problem is on appropriate condition in using each tunneling models in examining molecular structure. Our work will clarify the conditions and limitations inherited in I-V analysis using these methods and provide a proper guide for the modeling methods.

This manuscript is organized as following. First, I-V plots were generated using three methods under different $\varepsilon_{0}$ and $\Gamma$, where we can observe the deviation of each equation from the other. We then compared $\varepsilon_{0}$ and $\Gamma$ extracted by fitting experimental results obtained from literature papers using the three Equations to evaluate the error level under different conditions. We certainly propose numerical recommended method in I-V analysis considering it adopts least theoretical approximation and showed the widest applicability, while the two methods (Eq 2 and Eq 3 ) have to be used under limited conditions. At last, we summarized a phase map of applicability of the three methods as a guide for their proper usage in charge transport study in molecular devices. 


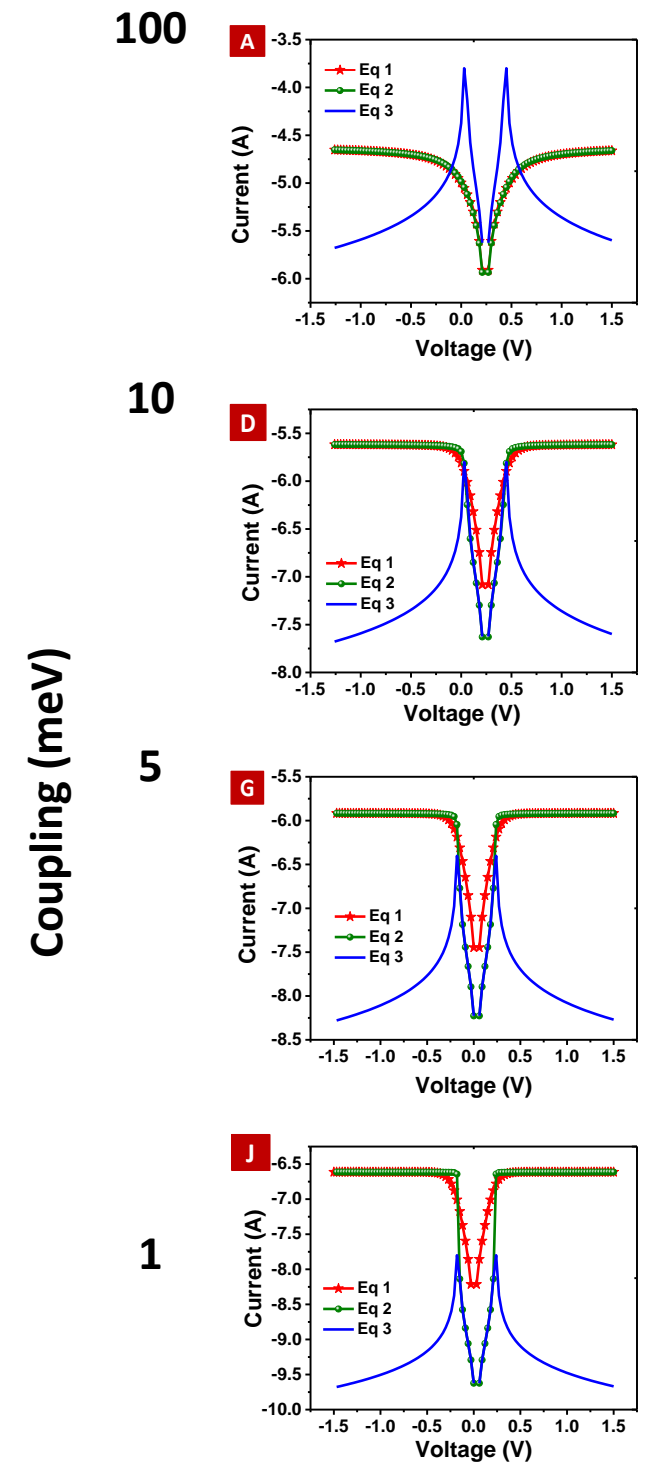

0.1
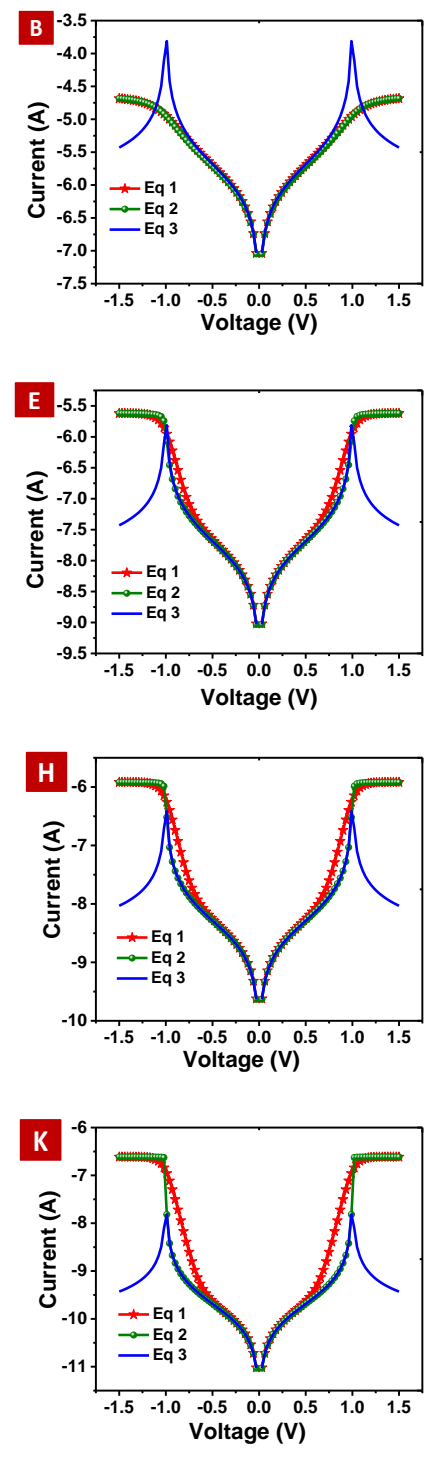

0.5
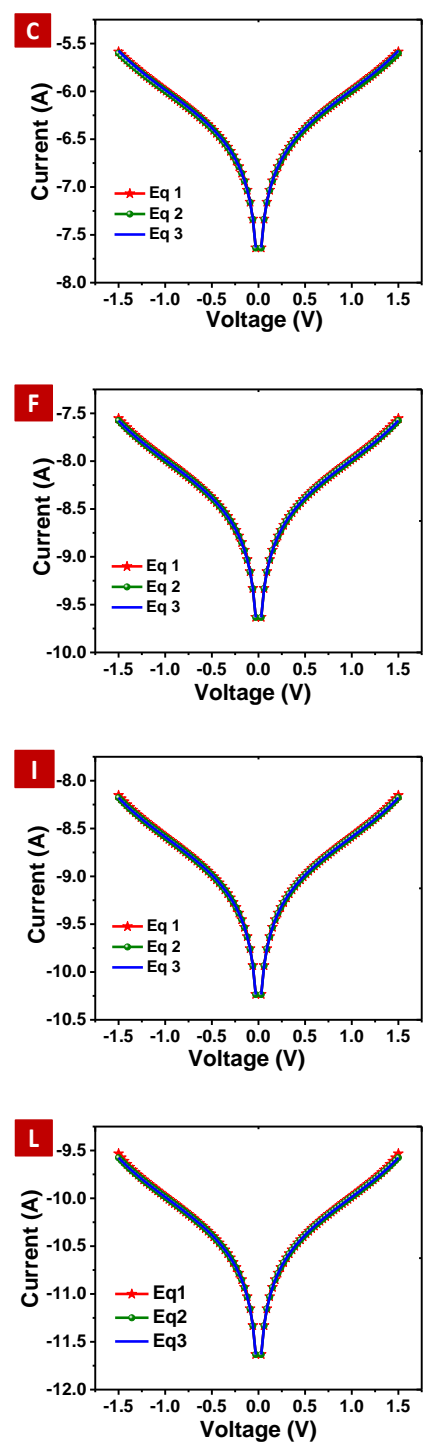

1

\section{Energy Barrier (eV)}

Figure 2: Current -Voltage (I-V) plots at different coupling and energy offset under single level model (SLM) by Eq 1, 2, and 3 
The tunneling transport through SLM can be describe by Landauer-Büttiker formalism (Eq 1)7, 10, 11 which shows relation of current with transmission probability $(\mathrm{Tr})$ that depends on energy offset and the coupling strength between electrodes and molecule. In the simulation, we consider the energy offsets of $1 \mathrm{eV}, 0.5 \mathrm{eV}$ and $0.1 \mathrm{eV}$, while coupling was set to be $100 \mathrm{meV}, 10 \mathrm{meV}, 5 \mathrm{meV}$ and $1 \mathrm{meV}$. These values were chosen based on experimental results reported in literatures. The quantum conductance was $2 \mathrm{e}^{2} /$ $h=77.4 \mu \mathrm{S}$, and voltage division factor $(\gamma)$ was 0.5 , i.e. symmetric situation. The bias range for the simulation is 1.5 $\mathrm{V}$ in both positive and negative polarities.

In method 1, the energy step for numerical integration was set to be $0.2 \mathrm{meV}$ and lower and upper limit of the integration is $-5 \mathrm{eV}$ and $5 \mathrm{eV}$. It should be emphasized that the integration step size should be significantly smaller than the width of the transmission peak, which is defined by the coupling parameter in Eq 1.3. We found this step size and integration limit are generally enough even down to the 1 meV coupling situation, and further smaller step size and wider integration range produce none noticeable difference in I-V plot. At last, the thermal energy $\left(\mathrm{K}_{B} \mathrm{~T}\right)$ in the Fermi function in Eq 1 was set to be $0.025 \mathrm{eV}$ for room temperature (298K).

In simulation of I-V response using analytical equations Eq 2 and $\mathrm{Eq} 3$ in comparison to numerical integration Eq 1, the same parameters were adopted i.e. coupling strength $(\Gamma)$, energy offset $\left(\varepsilon_{0}\right)$ and the bias voltage (V) window. Plotting $\mathrm{I}-\mathrm{V}$ data provides an overview of the general appearance of the I-V curves and how the shape of the curves differ from each other at different transport parameters using the above three tunneling equations.

The fitting were performed first, by extracting data from experimental I-V curves of corresponding literatures using Engauge Digitizer software and the I-V plot was digitized into 501 points. ${ }^{31}$ Then, after mathematical description of all code segments or/ parameters, the acquired data was fitted via nonlinear fitting regression in MATLAB software using three tunneling equations one at a time, and the initial guess values of energy offsets and coupling were adjusted during fitting until the best fit was obtained. According to the explanation above, for the numerical integration, the sept size should be significantly smaller than the coupling. Therefore if coupling is smaller than 1 meV, smaller step should be adopted until no significant difference can be observed.

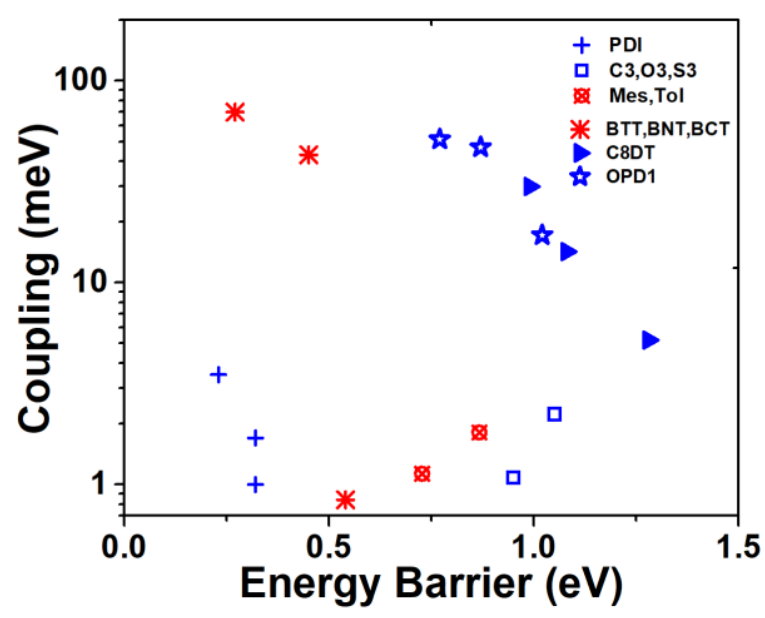

Figure 3. Summarized value of coupling as function of energy offset taken from literature.

\section{$0.3 \quad$ Results and discussion \\ 0.3.1 I-V plot characteristics}

To explore the scope of applying single level tunneling models, we first studied I-V characteristics under different coupling and energy offset conditions (figure 2) using the three methods, as summarized in figure 5 . We can see the $\mathrm{I}-\mathrm{V}$ characteristics by the three modeling methods are significantly dependent on coupling and energy offset. First, when coupling is relatively strong, $100 \mathrm{meV}$, Eq 1 and Eq 2 agree with each other very well, while Eq 3 does not work for small energy offset as low as $0.1 \mathrm{eV}$. When barrier increases to $0.5 \mathrm{eV}$, Eq 3 gradually conform and works only under low bias i.e. far from resonance. As the energy offset increase to $1 \mathrm{eV}$, Eq 3 fully worked at this energy offset at all provided bias range (Figure $2 \mathrm{~A}-\mathrm{C}$ ). Clearly, the working bias range of Eq 3 depends on the barrier height, the larger energy offset is, the larger bias range that Eq 3 can works. When coupling strength decrease to $10 \mathrm{meV}$ and $5 \mathrm{meV}$ (mild coupling strength, Figure $2 \mathrm{D}-\mathrm{F}$ and G-I), Eq 2 starts to deviate from Eq 1. This is because the broadening of Fermi distribution under room temperature ( $25 \mathrm{meV}$ ) become comparable to the coupling strength, so that the step function condition for Eq 2 will incur more and more error. Eq 3 failed to work again at low barrier and partially work at the mild barrier height $(0.5 \mathrm{eV})$. At a very weak coupling strength ( $1 \mathrm{meV})$, again Eq 1 can reasonably describes the tunneling process while Eq 2 and Eq 3 continue to work effectively at high barrier. In this situation, the Fermi distribution cannot be reduced to a step function as the precondition for Eq 2. Therefore, neither the conditions of Eq 2 nor Eq 3 can be satisfied (as seen in figure $2 \mathrm{~J}$ and $\mathrm{K}$ ). On contrary, the validity of these two analytical expressions 
at high barrier is contingent to a very small integration step size $(\leq 0.2 \mathrm{meV})$ of Eq 1 that should be smaller than the width of the transmission peak (figure $2 \mathrm{~L}$ ).

\subsubsection{Re-modeling experimental results}

In order to elaborate our idea on the limit of applicability of the three SLM modeling methods and their difference, we re-examined their modeling performance on experimental results from published literature papers. Figure 3 summarized the reported fitting parameters, coupling strength and energy offset, of the selected literature ${ }^{13,17,18 \text {, }}$ 24,32 , which provided general overview of the range of the reported values. The data presented below are acquired from their originate papers whereby those in red are obtained by using Eq 1 and for those appear in blue only coupling was obtained by Eq 3 while energy offset was from transition voltage (TVS). From the graph the data accommodated below $10 \mathrm{meV}$ under mild and low energy offsets are vulnerable to inaccuracy according to our discussion above, which indicated the application of Eq 3 is quite limited at low coupling and energy offset (energy barrier). We next try to redo the model fitting on the experimental results using the three methods (Eq 1 to 3 ) and compare the extracted parameters with the values from the original paper to check their performance and reliability.

We first check the results from Xie et al. ${ }^{18,32}$ in their work, Xie and coworkers first find the energy offset $\varepsilon_{o}$ from transition voltage $\left(V_{t}\right)$ using following equation.

$e V t=2 \varepsilon_{o} / \sqrt{3}$
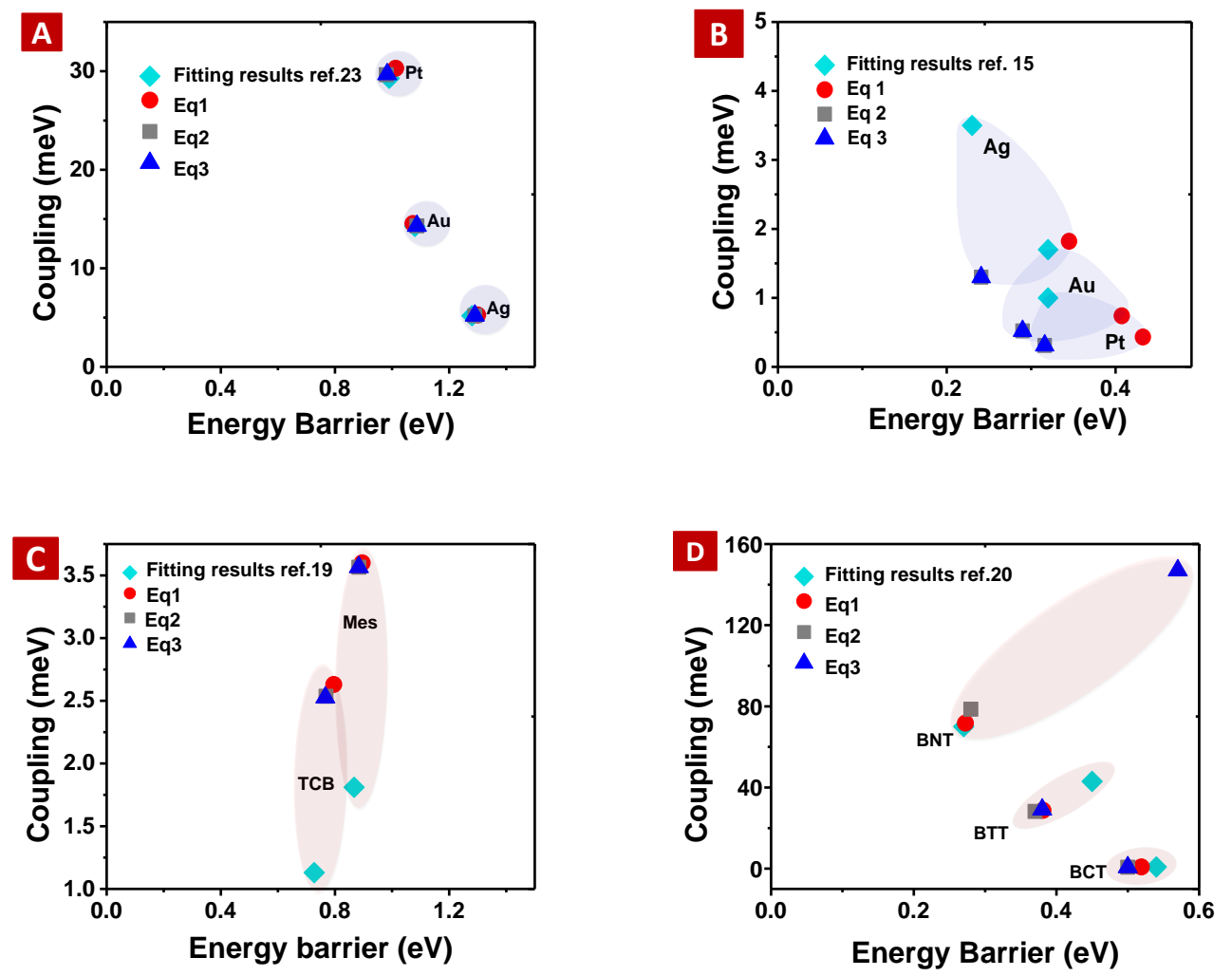

Figure 4: A comparison of individual fitting results of the three tunneling models with literature reported results for a) Octane dithiols (C8DT) on $\mathrm{Ag}, \mathrm{Au}, \mathrm{Pt}^{23}$ b). Isocyanide terminated perylene diimide $\left(\mathrm{CN}_{2} \mathrm{PDI}\right)^{32}$ (c) Mesitylene (Mes) and 1, 2, 4-trichlorobenzene (TCB) ${ }^{17}$ d). 4 , 4bisnitrotolane (BNT), 4, 4-bisthiotolane (BTT) and 4, 4-biscyanotolane (BCT) ${ }^{24}$ 
Then they use zero bias conductance (G) and effective number of molecules that contribute to transport in the junction to find the coupling using following equation:

$G=N G_{o} \frac{\Gamma^{2}}{\varepsilon_{o}^{2}}$

Figure $4 a$ and $b$ are the plots of extracted coupling strength and energy offset using the three methods as mentioned above (fitted I-V curves can be found in supporting information figure S1), and Xie's results. These results are also summarized in Table 1 . It can be seen that the C8DT molecular junctions (figure 4a) had high energy offset and the coupling strength of ascends from $\mathrm{Ag}, \mathrm{Au}$ and $\mathrm{Pt}$. The results of both tunneling equations and literature are well coincided supporting the explanation above on similarity in $\mathrm{I}-\mathrm{V}$ behavior for both three models under high barrier height. On the other hand, contemplating figure $4 b$, the molecular junction exhibited low coupling and energy offset (see fitting I-V curves in supporting information figure S4). Generally, under this condition the I-V curves of Eq 1 behaves different from that of Eq 2 and Eq 3 and the fitting results is also expected to vary because at this condition the applicability of Eq 2 and Eq 3 is highly limited by the small barrier and coupling strength. Therefore, Eq 2 and Eq 3 are not accurate enough any longer to be used and indeed we can see clear difference between the results from Eq 1 relative to Eq 2 and Eq 3 . The reported results from Smith and Xie et al in ref 15 deviated from both two sides because they adopted the hybrid method of TVS plus Eq 3 as described above, which under estimated the energy barrier compared to Eq 1 and overestimated the coupling compared to all the three methods.

We next turn to check the literature results of those fitted based on Eq 1. Figure 4c is the study done by Luka-Guth et al $^{17}$ who studied the role played by the solvent in the transport of the single molecular junction. This molecular junction exhibits high energy offset and low coupling strength, similar to C8DT molecular junction (figure 4a). The fitting results are quite the same for all the three methods, which is reasonable for the high energy offset situation. However, for the coupling, our fitting results are significantly higher than the literature results. This spotted difference could is possibly related to the influence of energy step size in the numerical integration. We believe Luka-Guth et al did not use small enough step size when performing numerical fitting in their model study (see supporting information figure $\mathrm{S} 6$ for the fitting using big integration step $\sim 0.032 \mathrm{eV}$ ). This obtained results in this situation emphasize on caution that should be taken on choosing energy step size for numerical simulation of tunneling transport. We propose a very small step sized as shown in section 0.2 .

On the other hand, the experimental work of Zotti et al, 4,4-biscyanotolane (BCT), 4,4-bisthiotolane(BTT) and 4,4 bisnitrotolane (BNT) molecular junctions ${ }^{24}$ showed mild and low energy offset, $\sim 0.45 \mathrm{eV}$ to $\sim 0.27 \mathrm{eV}$ depending on the anchoring group (figurer $4 \mathrm{~d}$ and $\mathrm{I}-\mathrm{V}$ shown in supporting information 57). The results observed in this study has shown clearly different between three models particularly on BNT molecular junction while no substantial difference for BTT and BCT molecular junctions. The larger difference displayed on BNT molecular junction in Eq 3 can be highly related to low value of energy offset as shown in table 1 . In the condition of low energy offset and high applied bias, the resonance effect emerges, which Eq 3 cannot account for (figure 2A). This behavior can significantly result to alter the value of extracted fitting parameters when performing numerical simulation as it violate the condition at which Eq 3 can be applied.

\subsubsection{Map development}

Based on I-V curves generated in figure 2 and the analysis of the experimental fitting results, we developed a map as shown by Figure 5 . This map provides a summary of conditions for applying Eq 2 and Eq 3 with respect to Eq 1, and highlights the following information: 1) the first modeling method (Eq 1 ) has the largest scope of applicability i.e., can be applied in all conditions to examine

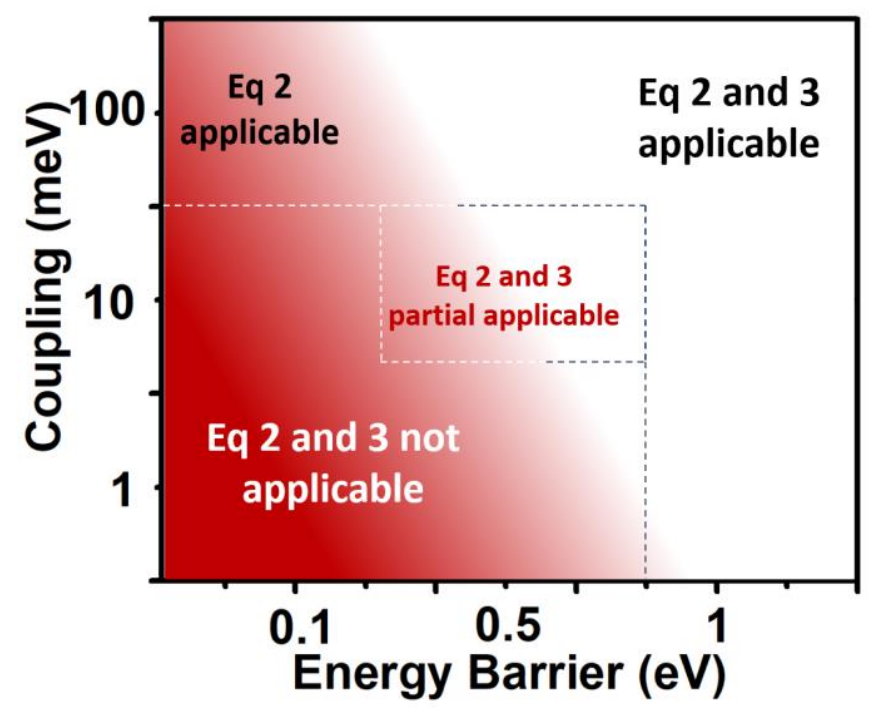

Figure 5: Scope of application of SLM Tunneling Equation 2 and Equation 3 deduced from I-V plot at different value of coupling strength and Energy offset at $1.5 \mathrm{~V}$ 
tunneling transport; 2) The scope of applicability of the other two methods (Eq 2 and Eq 3) is jointly restricted by the potential barrier and the coupling strength. We categorize our developed map in four sections. These categories are based on extent at which extracted fitting parameters varies and the difference in I-V response of the three methods. First, Eq 2 and Eq 3 did not work when coupling strength is very low (below $1 \mathrm{meV}$ ) at mild and low energy offset, we have seen from figure 2 that at low coupling strength there is pronounced difference between analytical expressions and Eq 1 which hinder their performance as molecular junction single level analysis model. However, this feature under mild energy offset deliberately vanishes when coupling increases. The variation between these expressions is so small that can be ignored. Likewise, further increase in coupling make Eq 1 and Eq 2 coincide meanwhile Eq 3 well behave under low bias far from resonance. On the other hand, Eq 3 failed to be applied at low energy offset regardless of coupling strength and we also observed this from BNT molecule fitting results of Zotti et al. On the contrary, Eq 2 works appropriately under this condition when coupling is relatively strong.
As we can see the application of Eq 3 is quite limited by coupling strength and energy offset, we strongly suggest high attention to be taken in analyzing experimental results based on it particular at low barrier. In addition, considering that Eq 3 is closely related to several other mathematical models, like transition voltage Spectroscopy (TVS) 26,27 and law of corresponding states $(\mathrm{LCS})^{33}$, it is very important to be aware of the condition and scope at which TVS and LCS can work effectively.

\subsubsection{The effect of number of molecules in the fitting}

In experimental studies, the number of molecules, or the number of channels, involved in charge transport is generally very hard to estimate. In single molecular junction like STM-BJ or MCBJ, the single molecule conductance was identified by statistical conductance peak. While in selfassembled monolayer (SAM) based junction, the active molecules under top electrode in the junction measurement can only be approximately estimated, which varied from $10^{2}$ to $10^{5}$ depending on the nature and contact geometry of the top electrode. In model fitting, the number of active molecules in the junction (or the channels) in the transport, was generally treated as a linear multiplication coefficient in front of the I-V response function. This is,

Table 1: Some of selected fitting Results using SLM tunneling expressions and literature reports

\begin{tabular}{|c|c|c|c|c|c|c|c|c|c|c|c|}
\hline results & $\Gamma(\mathrm{meV})$ & 5.19 & 29.93 & 3.55 & 9.79 & 2.42 & 6.08 & 43 & 70 & 1.81 & 1.13 \\
\hline Eq1 & $\Gamma(\mathrm{meV})$ & 5.29 & 30.3 & 3.9 & 10.0 & 2.6 & 6.8 & 28.73 & 71.7 & 3.600 & 2.63 \\
\hline & $\varepsilon_{\mathrm{o}}(\mathrm{eV}$ & 1.29 & 0.99 & 1.34 & 1.1 & 1.35 & 1.15 & 0.37 & 0.28 & 0.88 & 0.768 \\
\hline Eq2 & $\Gamma(\mathrm{meV})$ & 5.2 & 30 & 3.9 & 9.9 & 2.52 & 6.73 & 28.2 & 78.6 & 3.6 & 2.5 \\
\hline \multicolumn{12}{|l|}{ Eq3 } \\
\hline & $\Gamma(\mathrm{meV})$ & 5.2 & 30 & 3.9 & 9.9 & 2.52 & 6.73 & 29.1 & 147 & 3.6 & 2.5 \\
\hline
\end{tabular}

\footnotetext{
a \& b Eq 1 and Eq 3 respectively used for fitting in original literature work
} 
apparently, a very rough approximation. The multimolecule effect in the junction were explored and it was found the conductance is not a simple linear function of the number of molecules and the interaction between molecules can make significant difference $34-36$ .Nevertheless, in model fitting, it is quite common to put the guesstimated number of the molecules in front of the model as multiplication coefficient, which are

$$
\begin{aligned}
& I=N \frac{2 e^{2}}{h} \int_{-\infty}^{\infty} d E D_{\varepsilon}(E) \frac{\Gamma_{L} \Gamma_{R}}{\Gamma_{L}+\Gamma_{R}}\left[f_{L}(E)-f_{R}(E)\right] \\
& I=N \frac{2 e^{2}}{h} \frac{\Gamma_{L} \Gamma_{L_{R}}}{\Gamma}\left(\tan ^{-1} \frac{\Delta \varepsilon+e V / 2}{\Gamma}-\tan ^{-1} \frac{\Delta \varepsilon-e V / 2}{\Gamma}\right)
\end{aligned}
$$

And

$I=N \frac{2 e^{2}}{h} \Gamma_{L} \Gamma_{R}\left(\frac{e V}{\Delta \varepsilon^{2}-\left(\frac{e V}{2}\right)^{2}}\right)$

Since the magnitude of the current, especially at low bias, see Eq 3, is mainly determined by coupling, number of molecules and coupling are thus convoluted. Therefore, in modeling, the accuracy of coupling largely influenced by the number of molecules used in the fitting. When explaining charge transport in molecular junction, coupling strength and number of molecules $(\mathrm{N})$ in the junctions are like twin sisters that are hard to separate, especially when using Eq 3 , where $\mathrm{N}$ and $\Gamma$ are all pre-factors of the expression. However, for Eq 1 and Eq 2, $\Gamma$ also influences the shape of the I-V in addition to the magnitude, to be more precisely the non-linearity of the I-V. Therefore, number of molecules $\mathrm{N}$ can be obtained, in principle, as an independent parameter, using Eq 1 and Eq 2 at large enough bias that reached the nonlinear part of the I-V. In this study, our I-V plots (figure 2) did not consider the effect of number of molecules or channel i.e. number of molecules/channel assumed or considered to be unit. Therefore, the misinterpretation of fitting results in this study can originate from mis-estimation of the number of molecules. However, our map in Figure 5 can still be used to examine whether proper parameters were obtained by using correct modeling methods since it was developed under idea single molecule condition.

\section{4}

\section{Conclusions}

Theoretical modeling is an important strategy to identify transport mechanism and extract key transport parameters i.e. coupling strength and energy offset for tunneling mechanism. Proper utilization of the modeling methods in current voltage (I-V) analysis is crucial for obtaining reliable results and conclusion. Our extensive study in this work revealed the limitation of the modeling methods depending on the transport conditions, and possible misinterpretation of the transport parameters if the method were misused. In view of the discussion made on mathematical model of tunneling transport based on single level system current voltage (I-V) analysis, we would like to suggest following ideas in the practice of using SLM.

a) The modeling methods for single level system have their own limitation in practice. It is important to use the map in figure 5 above as the guidance for the applicability of Eq 2 and Eq 3.

b) Numerical integration Landauer formula (Eq 1) should provide more reasonable results relative to Eq 2 and 3. We would like to recommend it as a more general method than Eq 2 and Eq 3 for the modeling study of molecular junction study. We have included the MATLAB code for the practical use of the method in the supporting information.

It is important to notice as well the limitation of the numerical integration of Eq 1. The single level is whatsoever a simplified model and it may fail to work for systems with multi transmission channel and complex transmission spectrum. On the other hand, Eq 1 neither took into consideration the effect of variation in the density of state with respect to the energy $E$, and it also neglected the effect of electrostatic field under bias, like the energy level polarization, which is the basis of current rectification, and stark effect, which can make coupling strength bias dependent ${ }^{37}$. Therefore, care must be taken as well when applying method 1. E dependent DOS and bias dependent coupling could be further incorporated into the modelling method as done by Liu and Neaton.

Moreover, temperature dependent tunneling transport can in principle be captured by Eq 1 since the Fermi function have included the temperature effect. Unfortunately, the experimental study of this effect is still very limited, and it is not easy to distinguish from hopping transport. We provided one example on the usage of Eq 1 to study temperature dependent tunneling in the supporting information.

At last, we would like to emphasize that modeling methods based on physical model always have to face the problem of balancing between practicability vs. accuracy. While pure mathematical analyzing method like parabolic approximation (Taylor expansion) ${ }^{38}$ or polynomial expansion ${ }^{39}$ that does not required pre condition of single level model may be very valuable in the transport study in properly applied.

\section{Conflicts of interest}

Authors declare no conflicts. 


\section{Acknowledgements}

This work was supported by the National Natural Science Foundation of China (21773169, 21872103, and 21973069), the National Key R\&D Program (2017YFA0204503 and 2016YFB0401100), and the PEIYANG Young Scholars Program of Tianjin University (2018XRX-0007). The authors particularly appreciate the cooperation of Prof. Zuoti Xie for his contribution in this work.

\section{References}

1. J. C. Cuevas and E. Scheer, World Scientific Publishing 2017, 15.

2. T. A. Su, M. Neupane, M. L. Steigerwald, L. Venkataraman and C. Nuckolls, Nat. Rev. Mater, 2016, 1, 16002.

3. D. Xiang, X. Wang, C. Jia, T. Lee and X. Guo, Chem Rev 2016, 116, 4318-4440.

4. A. Troisi and M. A. Ratner, Small, 2006, 2, 172 181.

5. A. Nitzan and M. A. Ratner, Science, 2003, 300, 1384-1389.

6. R. L. McCreery, H. Yan and A. J. Bergren, Phys. Chem. Chem. Phys, 2013, 15, 1065-1081.

7. S. E. Tschudi and M. G. Reuter, Nanotechnology, 2016, 27, 425203.

8. A. R. Y. Garrigues, L. Wang, L. Singh, S. Del Barco, E. Nijhuis, C. A., Dalton Trans, 2016, 45, 1715317159.

9. A. R. Garrigues, L. Yuan, L. Wang, E. R. Mucciolo, D. Thompon, E. Del Barco and C. A. Nijhuis, Sci Rep, 2016, 6, 26517.

10. S. Datta, Cambridge University Press 2005.

11. X. Song, B. Han, X. Yu and W. Hu, Chemical Physics, 2020, 528, 110514.

12. A. M. a. A. Nitzan, ACS Nano Lett, 2011, DOI: doi/10.1021/nn202206e.

13. Z. Xie, I. Baldea, S. Oram, C. E. Smith and C. D. Frisbie, ACS Nano, 2017, 11, 569-578.

14. C. A. Nijhuis, W. F. Reus, J. R. Barber, M. D. Dickey and G. M. Whitesides, Nano Lett, 2010, 10, 36113619.

15. C. W. Beenakker, Phys Rev B Condens Matter, 1991, 44, 1646-1656.

16. J. P. Park, Abhay N.; Goldsmith, Jonas I.; Chang, Connie; Yaish, Yuval; Petta, Jason R.; Rinkoski, Marie; Sethna, James P.; Abruña, Héctor D.; McEuen, Paul L.; Ralph, Daniel C., Nature, 2002, 417, 722-725.

17. K. Luka-Guth, S. Hambsch, A. Bloch, P. Ehrenreich, B. M. Briechle, F. Kilibarda, T. Sendler, D. Sysoiev, T. Huhn, A. Erbe and E. Scheer, Beilstein J. Nanotechnol, 2016, 7, 1055-1067.

18. Z. Xie, I. Baldea and C. D. Frisbie, J. Am. Chem. Soc, 2019, 141, 3670-3681.
19. M. Souto, L. Yuan, D. C. Morales, L. Jiang, I. Ratera, C. A. Nijhuis and J. Veciana, J. Am. Chem. Soc, 2017, 139, 4262-4265.

20. R. C. C. Emily A. Weiss, George K. Kaufman,Jennah K. Kriebel, and M. D. Zhefeng Li, Maria A. Rampi, and George M. Whitesides, ACS Appl Mater Interfaces, 2007.

21. J. Chen, T. Markussen and K. S. Thygesen, J. Phys. $\operatorname{Rev} B, 2010,82$.

22. I. Bâldea, J. Phys. Rev B, 2012, 85, 035442.

23. Z. Xie, I. Baldea and C. D. Frisbie, J. Am. Chem. Soc, 2019, 141, 18182-18192.

24. L. A. Zotti, T. Kirchner, J. C. Cuevas, F. Pauly, T. Huhn, E. Scheer and A. Erbe, Small, 2010, 6, 15291535.

25. E. Lçrtscher, C. J. Cho, M. Mayo, M. Tschudy, M. Rettner and H. Riel, Chemphyschem, 2011, 12, 1677- 1682 .

26. I. Bâldea, Chemical Physics, 2010, 377, 15-20.

27. Beebe JM, Kim B, Frisbie CD and K. JG, ACS Nano, 2008, 2, 827-832.

28. Z. Xie, I. Baldea, C. E. Smit, Y. Wu and C. D. Frisbie, J. Am. Chem. Soc, 2015, 9, 8022-8036.

29 Z. X. Quyen Van Nguyen, and C. Daniel Frisbie, J. Phys. Chem C, 2021, 125, 4292-4298.

30. S.-Y. J. Pramod Reddy, Rachel A. Segalman,Arun Majumdar, Science, 2007, 315 1568-1571.

31. B. M. Mark Mitchell, Tobias Winchen and Antonio Trande, Engauge-Digitizer Software, http://markummitchell.github.io/engaugedigitizer, DOI: 10.5281/zenode.3941227).

32. C. E. Smith, Z. Xie, I. Baldea and C. D. Frisbie, Nanoscale, 2018, 10, 964-975.

33. I. Baldea, Z. Xie and C. D. Frisbie, Nanoscale, 2015, 7, 10465-10471.

34. G. C. S. Matthew G. Reuter, Thorsten Hansen,z Tamar Seideman, and Mark A. Ratner, J. Phys. Chem. Lett, 2011, 2, 1667-1671.

35. T. J. J. Trasobares J. Rech, T. Martin,O. Aleveque, E. Levillain,V. Diez-Cabanes,Y. Olivier,J. Cornil, J. P. Nys, R. Sivakumarasamy, K. Smaali, P. Leclere, A. Fujiwara, D. Théron, D. Vuillaume, and N. Clément, Nano Lett, 2017, 17, 3215-3224.

36. V. Obersteiner, G. Huhs, N. Papior and E. Zojer, Nano Lett, 2017, 17, 7350-7357.

$37 . \quad$ Z.-F. L. a. J. B. Neaton, Physical Chemistry C, 2017, 121, 21136-21144.

38. D. C. A. Vilan, and E. Kraisler, J. Am. Chem. Soc, 2013, 7, 695-706.

39. A. Vilan, J. Am. Chem. Soc, 2006, 111, 4431-4444. 